\title{
A primer of deuterium in drug design
}

\author{
Sarah Cargnin ${ }^{1}$, Marta Serafini ${ }^{1}$ \& Tracey Pirali $*, 1$ \\ ${ }^{1}$ Department of Pharmaceutical Sciences, Università del Piemonte Orientale, Largo Donegani 2, 28100 Novara, Italy \\ *Author for correspondence: tracey.pirali@uniupo.it \\ “Thanks to its high complementarity with this halogen in reducing drug attrition rate, we believe \\ that $\mathrm{D}$ will soon gain the attention it deserves"
}

First draft submitted: 12 June 2019; Accepted for publication: 14 June 2019; Published online:

20 September 2019

\section{Deuterium in medicinal chemistry}

Very recently, deuterium (D), the heavy and nonradioactive isotope of hydrogen $(\mathrm{H})$, has been a protagonist of cosmochemistry. Measurements of $\mathrm{D} / \mathrm{H}$ isotopic ratio of water in minerals collected by a mission from the asteroid Itokawa have finally demonstrated that the composition of water is indistinguishable from that in terrestrial rocks, suggesting that asteroids could have been a potential source of water during the formation of Earth [1]. Cosmochemistry is not the only field where $\mathrm{D}$ has recently gained popularity. Indeed, this isotope is appealing also to medicinal chemists as a means of improving existing drugs or as a tool in the design of new lead compounds. This is particularly true since 2017, where the USFDA approved the first deuterated drug, deutetrabenazine [2]. A comprehensive review on medicinal chemistry of $\mathrm{D}$ has been recently published [3]. In the present commentary, we wish to critically highlight the key advantages of $\mathrm{D}$ and review the most recent advances in clinics with deuterated compounds. Indeed, this is a flourishing field, with a plethora of clinical trials being pursued.

There is a sound reason for the success of this isotope in drug design: the D kinetic isotope effect. Due to the twofold higher mass of $\mathrm{D}$ compared with $\mathrm{H}$, the $\mathrm{C}-\mathrm{D}$ bond is much more resistant toward oxidative processes, for example catalyzed by CYP450 or by other enzymes involved in metabolism (for example; MAO, aldehyde oxidase), while retaining very similar steric properties. Such resistance must be proved nonetheless experimentally, case by case, as it is heavily dependent on the enzyme catalysis scenario and is more pronounced in those cases where the corresponding $\mathrm{C}-\mathrm{H}$ bond cleavage is the rate-limiting step.

Therefore, the H-D isosteric replacement in correspondence of an oxidizable soft-spot usually retains the pharmacodynamics, while improving the pharmacokinetics of a drug with a repercussion on half-life and/or of area under the curve values and, ultimately, on dose and/or dosing regimen. [4] The most striking example of this improvement is given by some inhibitors of aurora kinase reported in 2011 where the judicious incorporation of the two classical isosteres of H, D and fluorine, causes a significant increase of systemic drug exposure, together with a decrease of clearance, in four different animal species [5]. Although many other examples could be presented to show the ability of $\mathrm{D}$ to improve the pharmacokinetics of drugs, such amelioration does not always occur, and many factors that can mask or even revert the effects of deuteration come into play.

$\mathrm{D}$ has implications that go beyond the mere increase of metabolic stability. For instance, deuteration can be capitalized upon to reduce toxicity, as demonstrated by many examples of deuteration that have followed over the years, including the recent report on the c-Met inhibitor JNJ38877605 [6]. Here, the soft-spot is the 2-position of quinoline ring which is extensively oxidized by aldehyde oxidase. While the classic H-F isosteric replacement [7] is not possible as it hampers the formation of a key $\mathrm{H}$ bond with a residue of the kinase, $\mathrm{D}$ incorporation on one hand retains the biological activity, and on the other improves the metabolic stability, thereby reducing the formation of a nephrotoxic metabolite and increases the oral exposure.

$\mathrm{D}$ incorporation not only affects enzyme-catalyzed reactions but also pure chemical processes, such as epimerization. Many drugs are still sold as racemates due to chemically unstable stereocenters that make the two enantiomers interconverting. When incorporated in correspondence of chiral centres, D might decrease the rate of atom abstraction and stabilize the configuration in favor of the preferred enantiomer (the so-called D enabled chiral switching), as reported for CC-122, a thalidomide derivative in clinical trials for hematological cancers and solid tumors [8]. In 
this case, the (-)-deuterated enantiomer is antitumorigenic, whereas the other enantiomer has no effect on tumor growth.

\section{Deuterated compounds in clinical development: an update}

As already mentioned, the first deuterated compound that has entered clinical practice is deutetrabenazine for the treatment of both choreas associated with Huntington's disease and tardive dyskinesia. This success has given industry confidence to invest in the development of deuterated drugs, and as a result, more than 20 deuterated drugs are currently in clinical development [3], with 6 of them (BMS-986165, AVP-786, RT001, ALK-001, donafenib, HC-1119) having reached Phase III clinical trials.

There is ferment in the field, certainly, and relevant progress in the clinical testing of deuterated drugs has been made in the last few months. In December 2018, a new deuterated drug, HC-1119, publicly entered the panorama of deuterated medicines in clinical development. HC-1119 is the deuterated analog of enzalutamide, a second-generation competitive inhibitor of the androgen receptor which is effective for the treatment of metastatic castration-resistant prostate cancer (mCRPC). Even if enzalutamide was shown to significantly prolong overall survival in $\mathrm{mCRPC}$ patients compared to placebo, a higher risk of seizure occurrence was detected in enzalutamide users [9]. Given that such toxicity was found to be dose dependent, a deuteration strategy was applied in order to improve enzalutamide's PK properties and potentially limit side effects. Since enzalutamide is metabolized to $N$ demethylenzalutamide mainly by CYP2C8 and CYP3A4/5, the $N-\mathrm{CH}_{3}$ moiety was substituted by $N-\mathrm{CD}_{3}$ in order to attenuate the $N$-demethylation pathway. As a result, HC-1119 was shown to have an ameliorated PK profile and a higher safety margin compared with its nondeuterated counterpart in different in vivo models [10,11]. Nowadays, five clinical trials for HC-1119 are registered on Clinicaltrials.gov. Three of these are Phase I, open-label, clinical trials aimed at evaluating the pharmacokinetic properties of HC-1119 in healthy volunteers (NCT03776968) or in patients affected by mCRPC (NCT03774056, NCT03778047). The remaining two studies are Phase III, multicenter, randomized, double-blind, clinical trials assessing the efficacy and safety of oral HC-1119 in mCRPC patients when compared with placebo (NCT03851640) or oral enzalutamide (NCT03850795). It should be noted that the use of enzalutamide as a direct comparator renders the study design deeply informative since it offers the chance of reliably assessing the value of the deuteration. The estimated dates of Phase III studies completions are reported to fall within 2021.

Recent progresses in the field also concern ALK-001 and RT-001, the deuterated analogs of two nutritional compounds, vitamin A and linoleic acid, respectively. As we extensively described elsewhere [3], in these two examples $\mathrm{D}$ is key to the mechanism of action.

In ALK-001 ( $d_{3}$-vitamin A), D at C20 position slows down dimerization and subsequent production of lipofuscin that is responsible for retinal degeneration [12]. For this reason, this compound reached a Phase II clinical trial in 2015 for the treatment of Stargardt's disease, a rare autosomal macular dystrophy commonly leading to blindness in young age (NCT02402660). In March 2019, a Phase II/III multicenter, randomized, double-masked, placebo-controlled study (NCT03845582) was initiated to test ALK-001 for the therapy of geographic atrophy (GA) secondary to age-related macular degeneration (AMD). GA, an advanced form of AMD, is characterized by atrophic lesions of the outer retina, in turn resulting in the progressive and irreversible loss of visual functions [13]. Even if GA and Stargardt's disease are recognized as distinct disorders, they share the accumulation of lipofuscin granules in the retinal pigment epithelium as one of the main pathogenic mechanisms. As for Strargardt's disease, there is no treatment authorized for GA and ALK-001 was hypothesized to represent a potential answer to unmet therapeutic needs in GA. The completion date of the clinical trial on GA is expected to be on 2022 and, as declared by the Sponsor, ALK001 will be tested also in other ophthalmological disorders, including intermediate dry-AMD, autosomal recessive retinitis pigmentosa and autosomal recessive cone-rod dystrophy [14].

The incorporation of $\mathrm{D}$ in correspondence to the bis-allylic methylene group in linoleic acid has led to the discovery of RT001 ( $d_{2}$-linoleic acid ethyl ester) that is able to reduce the progress of lipid peroxidation [15]. Thanks to this peculiarity, it has entered the clinical development in 2015 for Friedrich's ataxia, a rare inherited neurodegenerative disorder associated with an increase in lipid peroxidation and characterized by areflexia, dysarthria and sensory deficits (NCT02445794). Three years later, a Phase II/III prospective open-label study was initiated to assess the efficacy and safety of RT001 in patients affected by infantile neuroaxonal dystrophy, a rare fatal neurodegenerative disorder characterized by a progressive loss of motor functions. On the basis of encouraging results seen in two Expanded Access patients, in February 2019 the Committee for Orphan Medical Products of 
the European Medicines Agency granted the Sponsor request for orphan medicinal product designation for the treatment of infantile neuroaxonal dystrophy with RT001 [16].

Lastly, BMS-986165 and CTP-543 stand out as the most intensively studied deuterated compounds in 2019. BMS-986165 is a de novo designed deuterated compound that acts as an inhibitor of tyrosine kinase 2 and that is even more potent and selective compared to the already marketed ones [17]. BMS-986165 entered clinical development in 2015 for the treatment of autoimmune disorders, including psoriasis, systemic lupus erythematosus and inflammatory bowel diseases. Ten new clinical trials for BMS-986165 have started since the beginning of 2019, of which one is an open-label, multi-center, Phase III study aimed at evaluating the efficacy and safety of BMS-986165 in patients affected by moderate-to-severe psoriasis (NCT03924427). Conversely, CTP-543 is the deuterated analog of ruxolitinib ( $d_{8}$.ruxolitinib), a JAK1 and JAK2 inhibitor, for which four clinical trials have started since March 2019 (Phase I: NCT03880136; Phase II: NCT03898479, NCT03941548, NCT03811912). These studies are expected to be concluded between 2019 and 2021.

Overall, the growing swarm of clinical trials on deuterated compounds clearly reflects the efforts made by the industrial world in unravelling the therapeutic potential of deuterated drugs. Yet, it must be acknowledged that for a medicinal chemist point of view clinical research is overly slow in feeding back information, with efficacy and safety data that are eagerly awaited to understand whether to pursue this strategy more often. Trials that will compare deuterated and non-deuterated compounds, in particular, will be highly informative.

\section{Concluding remarks}

D substitution is the smallest possible structural change that can be made to a molecule and it follows that its incorporation appears to be particularly useful for those soft positions that do not tolerate changes in steric hindrance or electronic properties. Despite the minimal change produced, it is becoming clear that D can provide significant advantages of different natures, according to each specific molecule, that go further beyond the simple prolongation of the half-life. It must be stressed that developing a deuterated drug is seemingly trivial, but is actually challenging and to be successful cannot disregard some concerns.

First of all, D incorporation does not solve issues for every compound and in every position. A judicious choice of both the molecule and the site of deuteration must be undertaken, exploring the metabolic fate and the enzymology of the given compound. This is necessary to enable an informed decision regarding the suitability of the approach. In this regard, it has been estimated that less than 10\% of all FDA-approved drugs are amenable to deuteration because of their chemical structure, or because they are metabolized in a way that D kinetic isotope effect would not be significant. Furthermore, potential metabolic switches (the deuteration of one position increases the metabolism at another site) can occur after deuteration, diverting the compound through other biotransformation routes. It follows that, if more than one oxidizable soft-spot is present, an initial per-deuteration of these positions is suggested, followed by progressive reversion to $\mathrm{H}$ until disappearance of the positive outcome given by $\mathrm{D}$.

A high attrition rate in the translation from in vitro to in vivo systems must be considered, especially in relation to the multiple factors that are not easy to be predicted and might hamper the correlation between in vitro and in vivo $\mathrm{D}$ kinetic isotope effect, including competing elimination mechanisms (for example Phase II reactions or urinary/biliary excretion of unchanged drug). Interspecies variability is an additional element of vulnerability that, conversely, can be foreseen at an early stage of development by careful evaluation of microsomal clearance values in microsomes of different species. In this regard, we would like to highlight a very recent paper that reports a MS/MS method that allows to simultaneously assess the metabolic stability toward liver microsomes of a 1:1 mixture of deuterated and nondeuterated compounds, with the advantage of being a rapid screening tool able to minimize experimental bias [18].

Finally, the synthesis of deuterated compounds is far from trivial: on one hand the deuterated pool strategy is limited by a narrow scope and relatively high cost of deuterated substrates, while on the other the exchange approach might suffer from low selectivity and $\mathrm{D}$ content, together with harsh and not always green experimental conditions [19]. It is therefore important to carefully evaluate from the very beginning the degree of D enrichment and selectivity that can be obtained, and maintained, during the process.

On these bases, the effects of deuteration incorporation are often unpredictable and the lack of obviousness for successfully patenting deuterated drugs comes from the unpredictability nature of such approaches. Many compounds when deuterated will not show a useful and significant difference compared with the precursor, and hence the demonstration of a positive outcome should be enough to satisfy this requirement. Nevertheless, patentability concerns are currently arising, with a variegate picture of allowed, rejected and allowed after restriction applications. 
This is somehow counterbalanced by the fact that, in economic and regulatory terms, an unquestionable edge of $\mathrm{D}$ switch is the possibility of faster and less costly clinical trials, that can partly capitalize on the extensive data previously collected on the nondeuterated version. Therefore, due to intellectual property issues, the era of D switching seems to be near to an ending, but $\mathrm{D}$ incorporation is currently establishing itself as a useful drug design strategy during de novo drug discovery, as demonstrated by the advent of BMS-98616. Besides that, as an important barrier to competition, claiming deuterated versions of new molecules in patent applications right from the start has already emerged as a trend for the large pharma companies, in some cases just as an elusive way to broaden the Markush structures, in others as a concrete result substantiated by examples and data.

In a very recent report on FDA drug approvals of 2018, fluorine has deserved nomination as the 'Atom of the Year' [20]. Thanks to its high complementarity with this halogen in reducing drug attrition rate, we believe that D will soon gain the attention it deserves. There are already several deuterated drugs in the pipeline, and it is foreseen that a number of them will follow the example of deutetrabenazine and hit the market in years to come.

\section{Financial \& competing interests disclosure}

The authors have no relevant affiliations or financial involvement with any organization or entity with a financial interest in or financial conflict with the subject matter or materials discussed in the manuscript. This includes employment, consultancies, honoraria, stock ownership or options, expert testimony, grants or patents received or pending, or royalties.

No writing assistance was utilized in the production of this manuscript.

\section{References}

1. Jin Z, Bose M. New clues to ancient water on Itokawa. Sci. Adv. 5(5), eaav8106 (2019).

2. Schmidt C. First deuterated drug approved. Nat. Biotechnol. 35, 493-494 (2017).

3. Pirali T, Serafini M, Cargnin S, Genazzani AA. Applications of Deuterium in medicinal chemistry. J. Med. Chem. 62(11), 5276-5297 (2019).

4. Gant TG. Using deuterium in drug discovery: leaving the label in the drug. J. Med. Chem. 57(9), 3595-3611 (2014).

5. Kerekes AD, Esposite SJ, Doll RJ et al. Aurora kinase inhibitors based on the imidazo[1,2-a]pyrazine core: fluorine and deuterium incorporation improve oral absorption and exposure. J. Med. Chem. 54(1), 201-210 (2011).

6. Zhan Z, Peng X, Sun Y et al. Evaluation of deuterium-labeled JNJ38877605: pharmacokinetic, metabolic, and in vivo antitumor profiles. Chem. Res. Toxicol. 31(11), 1213-1218 (2018).

7. Meanwell NA. Fluorine and fluorinated motifs in the design and application of bioisosteres for drug design. J. Med. Chem. 61(14), 5822-5880 (2018).

8. Jacques V, Czarnik AW, Judge TM et al. Differentiation of antiinflammatory and antitumorigenic properties of stabilized enantiomers of thalidomide analogs. Proc. Natl Acad. Sci. USA 112(12), E1471-E1479 (2015).

9. Scher HI, Fizazi K, Saad F et al. Increased survival with enzalutamide in prostate cancer after chemotherapy. N. Engl. J. Med. 367(13), 1187-1197 (2012).

10. Pang X, Peng L, Chen Y. Effect of N-methyl deuteration on pharmacokinetics and pharmacodynamics of enzalutamide. J. Labelled Comp. Radiopharm. 60(9), 401-409 (2017).

11. Jiang J, Pang X, Li L et al. Effect of N-methyl deuteration on metabolism and pharmacokinetics of enzalutamide. Drug Des. Devel. Ther. 10, 2181-2191 (2016).

12. Kaufman Y, Ma L, Washington I. Deuterium enrichment of vitamin A at the C20 position slows the formation of detrimental vitamin A dimers in wild-type rodents. J. Biol. Chem. 286(10), 7958-7965 (2011).

13. Fleckenstein $\mathrm{M}$, Mitchell $\mathrm{P}$, Freund $\mathrm{KB}$ et al. The progression of geographic atrophy secondary to age-related macular degeneration. Ophthalmology 125(3), 369-390 (2018).

14. Alkeus Pharma’s Clinical Trials. http://www.alkeuspharma.com/trials.html

15. Zesiewicz T, Heerinckx F, De Jager R et al. Randomized, clinical trial of RT001: Early signals of efficacy in Friedreich's ataxia. Mov. Disord. 33(6), 1000-1005 (2018).

16. EU/3/19/2134 | European Medicines Agency. https://www.ema.europa.eu/en/medicines/human/orphan-designations/eu3192134

17. Papp K, Gordon K, Thaci D et al. Phase II trial of selective tyrosine kinase 2 inhibition in psoriasis. N. Engl. J. Med. 379, 1313-1321 (2018).

18. Murphy RB, Wyatt NA, Fraser BH et al. A rapid MS/MS method to assess the deuterium kinetic isotope effect and associated improvement in the metabolic stability of deuterated biological and pharmacological molecules as applied to an imaging agent. Anal. Chim. Acta 1064, 65-70 (2019).

19. Atzrodt J, Derdau V, Fey T, Zimmermann J. The renaissance of H/D exchange. Angew. Chem. Int. Ed. Engl. 46(41), 7744-7765 (2007).

20. G de la Torre B, Albericio F. The pharmaceutical industry in 2018. An analysis of FDA drug approvals from the perspective of molecules. Molecules 24(4), pii:E809 (2019). 